\title{
GLAD!
}

Revue sur le langage, le genre, les sexualités

06 | 2019

Varia

\section{Luca Greco. 2018. Dans les coulisses du genre : la fabrique de soi chez les Drag Kings}

\section{Sara Garbagnoli}

\section{OpenEdition}

Journals

Édition électronique

URL : http://journals.openedition.org/glad/1813

DOI : $10.4000 /$ glad. 1813

ISSN : 2551-0819

Éditeur

Association GSL

Référence électronique

Sara Garbagnoli, «Luca Greco. 2018. Dans les coulisses du genre : la fabrique de soi chez les Drag Kings », GLAD! [En ligne], 06 | 2019, mis en ligne le 01 juillet 2019, consulté le 17 décembre 2020. URL http://journals.openedition.org/glad/1813; DOI : https://doi.org/10.4000/glad.1813

Ce document a été généré automatiquement le 17 décembre 2020.

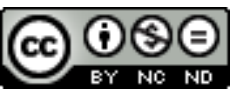

La revue GLAD! est mise à disposition selon les termes de la Licence Creative Commons Attribution Pas d'Utilisation Commerciale - Pas de Modification 4.0 International. 


\title{
Luca Greco. 2018. Dans les coulisses du genre : la fabrique de soi chez les Drag Kings
}

\author{
Sara Garbagnoli
}

\section{RÉFÉRENCE}

Luca Greco. 2018. Dans les coulisses du genre : la fabrique de soi chez les Drag Kings, Limoges : Lambert-Lucas. 176 pages.

1 Le titre de l'ouvrage de Luca Greco, Dans les coulisses du genre: la fabrique de soi chez les Drag Kings, recèle plus d'une vertu. Il est un hommage explicite au magistral travail du sociologue Erving Goffman dont Luca Greco ravive la démarche interactionniste en la faisant dialoguer de manière féconde avec la théorie butlerienne du genre comme dispositif d'inculcation dans les corps des normes régissant l'ordre sexuel. Il condense, ensuite, les principaux axes du projet intellectuel qui anime la monographie et, plus en général, le travail de l'auteur/e : étudier comment le genre et le langage s'imbriquent dans la construction des subjectivités et montrer les modalités à travers lesquelles leur politisation par des groupes minoritaires ouvre pour ces derniers un espace de manœuvre pour déplacer et pour tordre - le mot queer dériverait de torquere - les frontières de l'ordre sexuel (entre le masculin et le féminin, entre les hommes et les femmes, entre le privé et le public). Le titre illustre aussi de manière saisissante les interrogations d'un nouveau domaine de recherche, les études sur le genre et le langage, que Luca Greco a grandement contribué à faire émerger en France et qui a troublé les présupposés et les acquis tant des sciences du langage que des études de genre.

2 L'explicitation des conséquences épistémiques de cette contamination disciplinaire fait l'objet d'un premier moment analytique de l'ouvrage. L'auteur/e y illustre les fondements de l'approche à partir duquel ul mène son enquête : langage et genre sont 
théorisés comme des structures sociales naturalisées, inscrites dans les corps, dans les catégories mentales et dans les divisions objectives du monde social, et elles se construisent mutuellement. Un tel positionnement théorique présuppose des changements radicaux dans les définitions mêmes du langage et du genre. D'une part, contre la croyance de la neutralité et de la transparence de la langue qui ne serait que le reflet du réel ou la superstructure d'un mode de production, l'auteur/e conçoit le langage comme un conglomérat multisémiotique réunissant expressions verbales, nonverbales (gestes, postures) et matérielles (objets) qui produit des effets performatifs sur le réel et notamment sur les corps des groupes opprimés. Plus spécifiquement, le langage opère vis-à-vis des minoritaires sexuel.le.s (les femmes, les personnes lgbtqi+) comme une arme à double tranchant. Il est, à la fois, un instrument qui les blesse et les meurtrit car dans sa forme même il prend pour acquis que ce qui fonde la société serait l'hétérosexualité - Luca Greco renoue ici avec l'analyse wittiguienne de la consubstantialité entre ordre linguistique et ordre sexuel -, et un " champ de bataille » - c'est le titre de la partie introductive de l'ouvrage - que les groupes minoritaires peuvent investir pour opérer des formes de resubjectivation et d'émancipation. D'autre part, dans une telle approche, le genre et la sexualité se trouvent elles-mêmes redéfinies et investies d'une façon inédite par le langage : le langage en est la «face cachée », pour reprendre le titre d'un ouvrage codirigé par Luca Greco et Natacha Chetcuti.

3 Le livre se développe, ensuite, en trois étapes qui permettent progressivement à l'auteur/e de déployer son arsenal analytique et sa démonstration scientifique. Dans un premier moment, Luca Greco entreprend avec finesse une sociohistoire des conceptions, des représentations et des pratiques reliées à la catégorie "Drag King ». Dans sa définition courante, cette dernière se réfère à des personnes généralement assignées «femmes" à leur naissance qui s'habillent et se présentent en tant qu'« hommes». Par l'attention portée à l'imbrication entre dimension symbolique et matérielle des rapports sociaux, on peut inscrire cette quête génétique dans le sillage d'une histoire sociale, culturelle et catégorielle de «l'homosexualité » (au sens large du terme) telle qu'elle a été inaugurée par les recherches de Michel Foucault et repensée par l'historien George Chauncey dans son Gay New York, 1890-1940. Elle dessine en filigrane une histoire discontinue, fragmentée et polysémique qui rend compte de l'émergence dans les subcultures lgbtqi+ du XXe siècle d'une pluralité, voire d'une prolifération d'identités (l'auteur/e parle d'« explosion catégorielle»). Construits de manière oppositionnelle par rapport aux taxinomies pathologisantes véhiculées par les savoirs normatifs (médecine, droit, psychanalyse), ces systèmes catégoriels font éclater la notion même d'«homosexualité ». De là, se dégage en filigrane l'histoire des pratiques «drag » qui ont investi différentes grandes villes (New York, San Francisco, Londres, Paris) et qui sont le résultat de filiations hybrides et hétéroclites : des bals gay et lesbiens " en travesti » à la stylisation polarisée des rôles butch/fem dans les couples lesbiens, des performances artistiques de "personnification " masculine aux pratiques de réappropriation des masculinités par des femmes. Dans un deuxième moment de l'analyse, l'auteur/e présente les conditions de l'enquête et les enjeux d'une ethnographie qui se veut faite "pour » et " avec » les personnes observées. La mise en avant de l'appartenance relative de l'enquêteur.se - une personne queer assignée " homme » à la naissance - à la minorité stigmatisée sur laquelle porte l'enquête et la constante négociation collective et interactive de sa place au sein de l'atelier s'inscrivent dans la perspective d'une remise en cause critique du principe de « rupture 
épistémologique » hérité de la tradition durkheimienne. L'observation de sa propre participation devient, ainsi, pour l'auteur/e le site à partir duquel ul formule ses questions de recherche (qu'est-ce que construire et incarner différentes corporalités masculines quand on adopte une posture critique vis-à-vis de l'hétéronormativité ? Quels sont les procédés que les participant.e.s inventent pour fabriquer leur "soi » masculin ? De quelle façon l'idéologie naturaliste peut-elle réapparaître de manière non surveillée dans des discours tenus par les participant.e.s à l'atelier?) et élabore ses éléments de réponse. L'analyse des pratiques et des techniques de "kinguing " dans une approche multidimensionnelle de la présentation de soi - c'est le troisième moment analytique de l'ouvrage - met ainsi à jour le caractère artificiel et naturalisé de la construction binaire $\mathrm{du}$ genre et le rôle constitutif du langage dans son accomplissement. L'action de "se kinguer" apparaît ainsi comme le résultat de performances dénaturalisant non seulement la notion de "masculinité naturelle", mais aussi de celle du corps comme déjà sexué (masculin ou féminin). Comme déjà le « corps - et le langage - lesbien » des œuvres de Monique Wittig, les corps des « Drag Kings » (et leur langage) affirment par leur existence que le langage n'est pas un miroir du réel et que le corps n'est pas une donnée anatomique déhistoricisée et désociologisée, une "base naturelle» sur laquelle on ajouterait des couches " artificielles », mais une construction hétérosexualisée et naturalisée. Le corps c'est de l'histoire incorporée, du collectif, de l'« intercorporel » (Luca Greco invente la notion en la calquant sur celle d'intertextualité) qui inscrit chaque corps dans un répertoire historique et politique de corporalités qui lui préexistent et qui sont traversées et définies par des hiérarchies de genre, de sexualité, de classe, de race. L'interrogation critique des dispositifs catégoriels menée au sein des ateliers « Drag Kings » et analysée dans le livre fait émerger par contraste ce qui se passe en hétérosexualité et confirme ce que des analyses féministes - je pense, notamment, au travail de Colette Guillaumin sur la catégorisation - ont démontré.

Nommer, désigner sexuellement, dire socialement de quelqu'un/e ou à quelqu'un/e qu'ul est un homme ou une femme "par nature ", par "déterminisme biologique " revient à lui assigner une place dans l'ordre social, à lui attribuer un système de limites sous forme d'attribution de légitimité ou d'illégitimité à faire, à dire, à penser. De la nomination découlent un effet d'assignation statutaire et la tendance à acquérir socialement par incorporation les capacités ou les incapacités a priori attribuées au groupe sexuel auquel l'individu est assigné.e. Capacités et incapacités acquises seront à posteriori socialement appréhendées - dans un processus d'inversion de cause à effet comme découlant d'une prétendue "nature sexuelle » du sujet. Le livre devient, ainsi, une invitation à penser que, en prenant conscience de la force et de la brutalité des structures sociales et langagières qui définissent le régime hétérosexuel, il est possible, même si de manière locale et située, de changer de main le pouvoir de nomination et d'imaginer l'inimaginable d'un monde sans genre. Dans une période marquée par de farouches attaques contre les études de genre, qui s'inscrivent dans des formes plus générales de grave précarisation de la démocratie, l'ouvrage de Luca Greco est la preuve vivante que la théorie critique n'est pas un luxe, mais une nécessité vitale. 
INDEX

Mots-clés : catégorisation, corps, performativité, Drag King

Keywords : categorization, body, performativity, Drag King

Thèmes : Actualités

\section{AUTEURS}

\section{SARA GARBAGNOLI}

Sociologue et féministe, Sara Garbagnoli est doctorante à l'Université Paris 3. Ses recherches portent sur la théorie féministe, l'analyse du discours et la sociologie des mouvements sociaux. Avec Massimo Prearo elle est l'auteure de La croisade " anti-genre ». Du Vatican aux Manif pour Tous (Textuel 2017). Avec Vincenza Perilli elle a codirigé Non si nasce donna. Percorsi del femminismo materialista (Alegre, 2013). Elle a récemment publié dans les revues About Gender et Religion and Gender et participé aux ouvrages Campagnes anti-genre en Europe : des mobilisations contre l'égalité (PUL, 2018) et Antiféminismes et masculinismes d'hier et d'aujourd'hui (PUF, 2019). 